\title{
Preparation of 3D-Porous Graphene Aerogel for High- Performance Anode of Lithium-Ion Batteries
}

\author{
Peng $\mathrm{Li}^{1-3}$, Guiyun $\mathrm{Yi}^{1-4 *}$, Zhengting Zhang ${ }^{1-3}$, Haiyang Fan ${ }^{1-3}$, Yuanfeng $\mathrm{Wu}^{1-3 *}$, Weiwei Kang ${ }^{1-3}$, \\ Xiuxiu Zhang ${ }^{1-3}$, Bing $\mathrm{Xu}^{1-3}$, Yulong Zhang ${ }^{1-3}$ and Qi Sun ${ }^{1-3}$ \\ ${ }^{1}$ College of Chemistry and Chemical Engineering, Henan Polytechnic University, China \\ ${ }^{2}$ Collaborative Innovation Center of Coal Work Safety of Henan Province, China \\ ${ }^{3}$ Henan Key Laboratory of Coal Green Conversion, Jiaozuo 454003, China \\ ${ }^{4}$ State Collaborative Innovation Center of Coal Work Safety and Clean-efficiency Utilization, China \\ *Corresponding author: Guiyun Yi, College of Chemistry and Chemical Engineering, Henan Polytechnic University, Henan Key \\ Laboratory of Coal Green Conversion and State Collaborative Innovation Center of Coal Work Safety and Clean-efficiency Utilization, \\ China
}

Yuanfeng Wu, College of Chemistry and Chemical Engineering, Henan Polytechnic University, Henan Key Laboratory of Coal Green Conversion, China

\section{ARTICLE INFO}

Received: 幽 September 06, 2021

Published: 蔧 September 16, 2021

Citation: Peng Li, Guiyun Yi, Zhengting Zhang, Haiyang Fan, Yuanfeng Wu, et al., Preparation of 3D-Porous Graphene Aerogel for High-Performance Anode of Lithium-Ion Batteries. Biomed J Sci \& Tech Res 38(5)-2021. BJSTR. MS.ID.006205.

\begin{abstract}
Herein, a novel graphene aerogel (GA) with three dimensional (3D) porous structure was successfully prepared via a simple hydrothermal method, which was used as the anode active materials of lithium-ion batteries. Four samples with different hydrothermal reaction time $(2 \mathrm{~h}, 6 \mathrm{~h}, 10 \mathrm{~h}$, and $14 \mathrm{~h})$ were comparably synthesized, and the electrochemical performance of all the synthesizes samples was measured. The porous electrode material was found to possess the highest specific capacity (113.8 $\mathrm{mA} \mathrm{h} \mathrm{g}^{-1}$ at $2 \mathrm{~A} \mathrm{~g}^{-1}$ ), the best rate capability and the most stable cycling performance (664.8 $\mathrm{mA} \mathrm{h} \mathrm{g}^{-1}$ capacity retention at $100 \mathrm{~mA} \mathrm{~g}^{-1}$ over 100 cycles). Such excellent electrochemical performance of the composite may be attributed to the rich porous and defective structure of graphene aerogels, which can offer more pathways for $\mathrm{Li}^{+}$ions transportation and make the diffusion resistance decreasingly.
\end{abstract}

Keywords: Graphene Aerogel; Porous Electrode Material; Lithium-Ion Batteries; Anode Material

\section{Introduction}

Energy is recognized as an important material basis as well as a guarantee for human survival and development all the time [1,2]. However, with the excessive use of coal, petroleum and natural gas, the ever-increasing energy shortage and environmental contamination had become two major challenging problems facing all global nations. Thus, to effectively address such critical issues, extensive effort has recently been devoted to exploring efficient and clean energy conversion applications, such as lithium ion, sodium ion and fuel batteries. As an excellent energy storage device, lithium ion battery (LIBs) has attracted worldwide concerned and has achieved commercialization. Due to the prestigious advantages of high power per unit mass, high cycle life and high safety performance, LIBs has been applied in a wide range of fields, such as in mobile phones, electric vehicles and aviation $[3,4]$.

The structural configuration of LIBs mainly consists of positive and negative electrode materials, a diaphragm, electrolyte, and current collector [5]. In a typical discharging process, the $\mathrm{Li}^{+}$ generated by the negative electrode is embedded into the positive electrode through the electrolyte and the electrons are collected by the current collector and move in the opposite direction of the 
external circuit [6]. While for charging, it is indeed the reverse process as described above. Thus, during the charging and discharging period, the more $\mathrm{Li}^{+}$ions moving between the positive and negative electrodes, the greater the activity being generated and the higher the charge-discharge specific capacity [7]. As a consequence, such principle of electrochemical conversion has confirmed that suitable anode materials are now playing pivotal role in improving electrochemical performance of LIBs.

As is well known, graphene, consisting of a single layer carbon atoms with $\mathrm{sp}^{2}$ bonding, is a typically two-dimensional carbon material with outstanding excellent electrical conductivity and mechanical strength $[8,9]$. Its theoretical capacity can reach up to $744 \mathrm{mAh} \mathrm{g}^{-1}$, which is twice as much as that of graphite. This may be attributed to the fact that both sides of a graphene sheet can accommodate two $\mathrm{Li}^{+}$ions, in each hexagonal loop of carbon $\left(\mathrm{Li}_{2} \mathrm{C}_{6}\right)$ [10]. Therefore, graphene is regarded as a potential candidate to substitute commercial graphite anodes and has been widely used in LIBs.

However, the powerful $\pi-\pi$ stacking interactions and Van Der Waals forces lead to graphene sheets easily self-aggregate, which significantly reduces the available specific surface area and increases $\mathrm{Li}^{+}$ion transmission resistance [11]. In order to solve the aggregation problem, researchers have devised a great deal of advanced strategies. In particular, self-assembling graphene nanosheets into GA is one of the most effective methods. For example, our previous work demonstrated that PS nanospheres were used as sacrificial templates to fabricated nitrogen-rich graphene aerogel, where PS microsphere intercalation prevented the accumulation of layers in the GO sheet [12]. The obtained GA exhibited excellent electrochemical performance due to the layered porous structure. In addition, Zhang [13] et al. successfully fabricated free-standing nitrogen-doped graphene aerogel as the anode material for sodium ion batteries and it showed a high cycling performance $\left(287.9 \mathrm{~mA} \mathrm{~h} \mathrm{~g}^{-1}\right.$ after 200 cycles at a current density of $100 \mathrm{~mA} \mathrm{~g}^{-1}$ ). In another similar work, Albarqouni [14] et al. successfully prepared rGO aerogel as supercapacitor electrode materials by utilizing the reduction capability of carbonic acid in soft drinks and enhanced charge storage capacity $\left(121 \mathrm{~F} \mathrm{~g}^{-1}\right.$ at 0.4 $\mathrm{A} \mathrm{g}^{-1}$ ). Thus, GA exhibit promising applications in electrochemistry field. However, to the best of our knowledge, works related to GA for LIBs are still limited.

In this work, GA used as the anode of LIBs were prepared by a facile hydrothermal strategy without any additives and templates. GA prevented the restacking of graphene nanosheets to enlarge the contact area between the electrolyte and the electrode, which simultaneously reduced ion and electron diffusion resistance [15]. Besides, it was feasible to tailor the oxygen-containing functional group and surface defects of GA through adjusting the hydrothermal synthesis time. Benefiting from their porous structure and large specific surface area, the prepared GA-based LIBs show outstanding cycling stability (664.8 $\mathrm{mAh} \mathrm{g}^{-1}$ at $0.1 \mathrm{~A} \mathrm{~g}^{-1}$ after 100 cycles) and superior rate capability, which demonstrates their potential application for $\mathrm{Li}^{+}$storage. The current research provides a valuable guidance for developing high-performance anode material for application in LIBs.

\section{Experimental}

\section{Materials and Chemicals}

All reagents were purchased commercially without further purification. GO was obtained by the method mentioned in our previously reported work [16].

\section{Materials Characterization}

To investigate the microstructure and morphology of the asprepared samples, they are examined by advanced characterization tools like X-ray diffraction (XRD, Bruker, Germany), ULTRA 55 scanning electron microscope (SEM, ZEISS, Germany) and Raman spectroscopy (LabRAM HR $800 \mathrm{UV}$ ), respectively. The functional groups and elemental analysis of all samples were evaluated by X-ray photoelectron spectra (XPS) on ESCALAB 250Xi (Thermo Scientific, USA).

\section{Electrochemical Measurements}

The mixture of active materials, carbon black and polyvinylidene fluoride (PVDF) (weight ratio = 8:1:1) was dissolved in N-methyl2-pyrrolidinone (NMP) solvent to prepare working electrodes. The assembling of 2016 coin-type cells was similar to that of reported previously. Cyclic voltammetry (CV) curves were recorded by a Shanghai Chenhua CHI 760e electrochemistry workstation in the range from $3.0 \mathrm{~V}$ to $0.01 \mathrm{~V}$ at $0.2 \mathrm{mV} \mathrm{s}^{-1}$. Furthermore, galvanostatic charge/discharge cycles were measured using a NEWARE CT-4008 battery testing system at a current density range from 0.05 to $2 \mathrm{~A}$ $\mathrm{g}^{-1}$ versus $\mathrm{Li} / \mathrm{Li}^{+}$.

\section{Results and Discussion}

\section{Structural Characterization}

Fig. 1(a) presents the preparation and lithium storage process of the GA. Firstly, $10 \mathrm{~mL}$ of GO ( $3 \mathrm{mg} \mathrm{mL}^{-1}$ ) was transferred to 25 $\mathrm{mL}$ stainless-steel autoclave and then was maintained $180{ }^{\circ} \mathrm{C}$ for $2,6,10$, and $14 \mathrm{~h}$. Subsequently, the GA were obtained by freezedrying and labeled as GA-2, GA-6, GA ${ }^{-10}$ and $\mathrm{GA}^{-14}$, respectively. The excellent $\mathrm{Li}^{+}$storage performance of GA-X electrode was due to the porous and defective structure of graphene aerogels, which can provide more $\mathrm{Li}^{+}$ions pathways and reduce diffusion resistance. In the process of hydrothermal reduction of graphene oxide, oxygen- 
containing functional groups and hydrogen bonds on the surface of graphene oxide promote cross-linking between the sheets, and the gaps are filled with water molecules [5,17]. As a result, the porous structure is preserved by a sublimation process in freeze drying.

Shown in Figure $1(b \sim k)$ are the SEM images of all samples in order to investigate their morphologies and microstructures.
Through low magnification (Figure $1(b, d, h$ and j)) and high magnification (Figure 1(c, e, i, and k)), all samples exhibit the typically hierarchical and interconnected framework structure consisting of twisted and cross-linked graphene nano-sheets, which can provide more channels for $\mathrm{Li}^{+}$ions transport and reduce electrons transport resistance, thereby enhancing the electrochemical performance of GA-X.
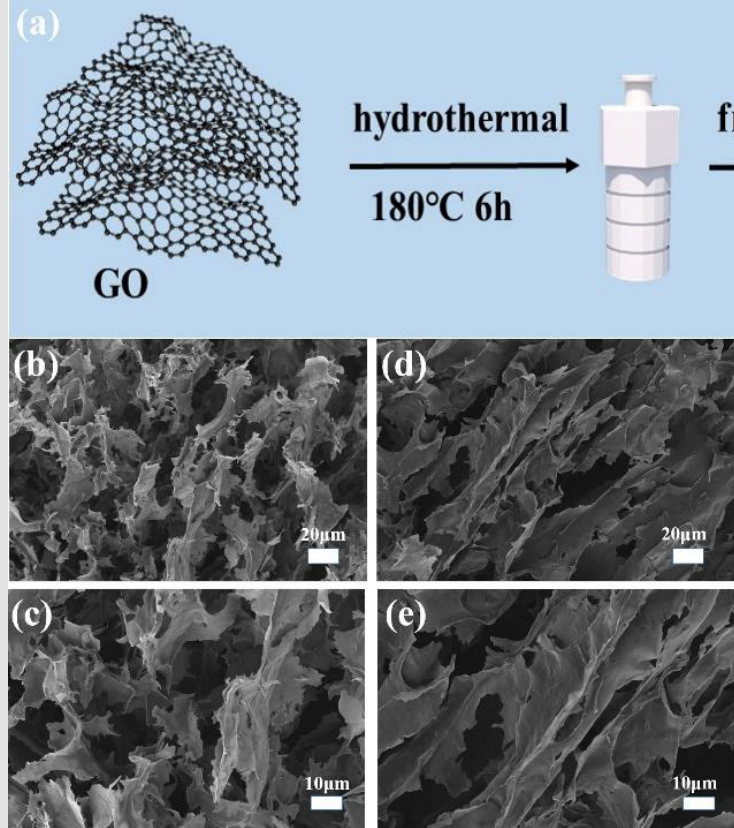
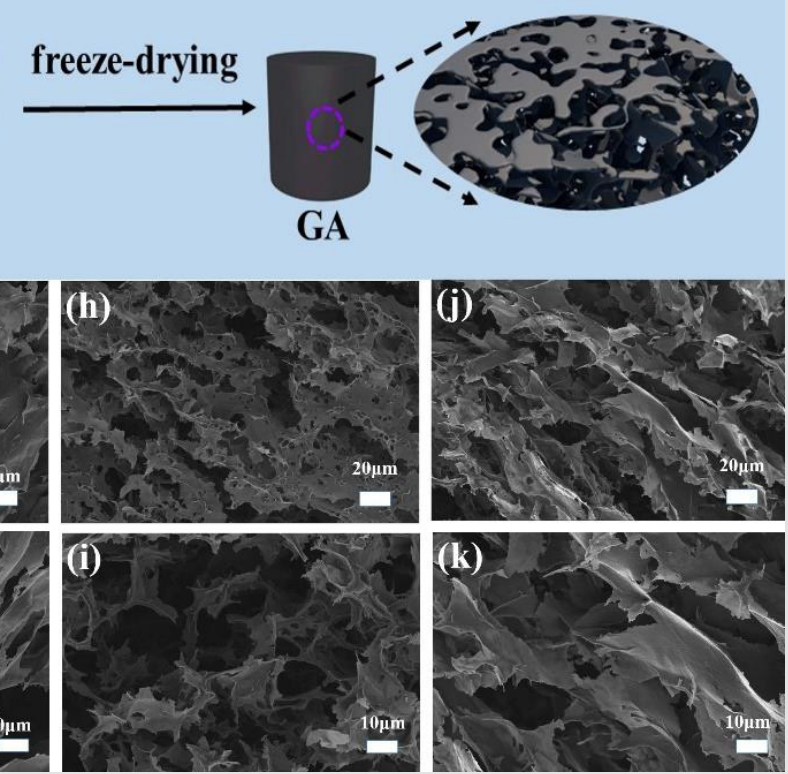

Figure 1: Schematic illustration of procedure to prepare GA anode and SEM images of GA-2(b, c), GA-6(d, e), GA-10(h,i) and GA-14(j,k).

To better clarify the structure variation of synthesized materials after the hydrogenation process, XRD, Raman and XPS measurements are performed. For comparison, Figure 2(a) shows the XRD patterns of graphite, GO, GA-2, GA-6, GA-10 and GA-14, respectively. It could be obviously observed that the characteristic peak of graphite appears at $26.3^{\circ}$, which corresponds to the interlamellar spacing of $0.348 \mathrm{~nm}[9,18]$. After the Hummers method of oxidation process, the sharp peak shifted to $10.6^{\circ}$, which could be assigned to the (002) reflection [19], and the interlamellar spacing was expanded to $0.838 \mathrm{~nm}$, which was caused by the presence of various oxygen-containing functional groups (hydroxyl, carboxyl, epoxy and carbonyl) and the introduction of $\mathrm{H}_{2} \mathrm{O}$ molecules [20]. More importantly, after hydrothermal treatment, the broad (002) diffraction angle and interlayer spacing of GA-2, GA-6, GA ${ }^{-10}$ and $\mathrm{GA}^{-14}$ gradually increases to $24.48^{\circ}(0.372 \mathrm{~nm}), 24.80^{\circ}(0.367 \mathrm{~nm})$, $24.94^{\circ}(0.365 \mathrm{~nm})$ and $25.14^{\circ}(0.362 \mathrm{~nm})$, respectively, which is attributed to the fact that the surface functional groups and intercalated $\mathrm{H}_{2} \mathrm{O}$ molecules gradually decrease. This confirms that the hydrothermal time exerts different degrees of reduction impact on graphene oxide.
As can be seen from Raman spectra of all samples in Figure 2(b), two characteristic peaks at about 1348 and $1583 \mathrm{~cm}^{-1}$ appear [21]. $\mathrm{G}$ band represents the stretching vibration of sp2-bonded carbon atoms of graphitic layers, and D band corresponds to vibrations of sp3-bonded carbon atoms of the defective graphitic structure or disorders [22]. Generally, The D/G peak intensity ratio (ID/IG) value was used to assess the disorder of graphitic materials [23,24]. ID/IG of the GO (0.97), GA-2 (1.21), GA-6 (1.22), GA-10 (1.52) and GA${ }^{14}$ (1.61) increase with the increment of hydrothermal time, which was due to the partial removal of the oxygen-containing functional groups on the graphene surface, indicating the high degree of reduction in the process of crosslinking graphene into aerogels.

X-ray photoelectron spectroscopy (XPS), a significant characterization tool to give direct information about the elemental composition and surface functional groups of the obtained samples, has been carried out for further analysis. Two obvious peaks at 285 and $532 \mathrm{eV}$ in Figure 2(c), which can be related to $\mathrm{C}$ and $\mathrm{O}$ elements, respectively [25]. More importantly, the atomic ratio of carbon and oxygen (C/O), is in the following order: GO (2.26) < GA-2 (4.52) 
$<$ GA-6 (4.76) (GA $\left.{ }^{-10} 4.73, \mathrm{GA}^{-14} 4.71\right)$, which indicated that the oxygen functional groups of GA successfully are decomposed with the hydrothermal reaction time and no significant change occurs after six hours (the detailed data was shown in Table 1). Based on in-depth analysis of the shark peak in high resolution C 1s XPS spectrum (Figure 2d), four types of $\mathrm{C}$ species can be detected in those samples. Furthermore, the peaks located at 290.9, 288.3, 285.8 and $284.3 \mathrm{eV}$ correspond to carboxyl groups (-COOR), carbonyl groups $(\mathrm{C}=0)$, epoxy groups $(\mathrm{C}-\mathrm{O}), \mathrm{sp}^{3}$ and $\mathrm{sp}^{2}$ carbon, respectively [26-28]. Shown in Fig. 2(e) is the high resolution 01s spectra, from which it can be seen that GA exhibits four peaks at the binding energy of $536.1,532.8,531.3$ and $530.1 \mathrm{eV}$, which are assigned as the $\mathrm{H}_{2} \mathrm{O}, \mathrm{HO}-\mathrm{C}=0, \mathrm{C}-\mathrm{O}-\mathrm{C}$ and $\mathrm{C}=0$, respectively $[21,29]$.

Table 1: Relative atomic percentages according to the XPS spectra.

\begin{tabular}{|c|c|c|c|}
\hline Samples & C1s(\%) & 01s(\%) & Atomic ratio C/0 \\
\hline GO & 69.3 & 30.7 & 2.26 \\
\hline GA-2 & 81.9 & 18.1 & 4.52 \\
\hline GA-6 & 82.6 & 17.4 & 4.76 \\
\hline GA-10 & 82.6 & 17.4 & 4.73 \\
\hline GA-14 & 82.5 & 17.5 & 4.71 \\
\hline
\end{tabular}
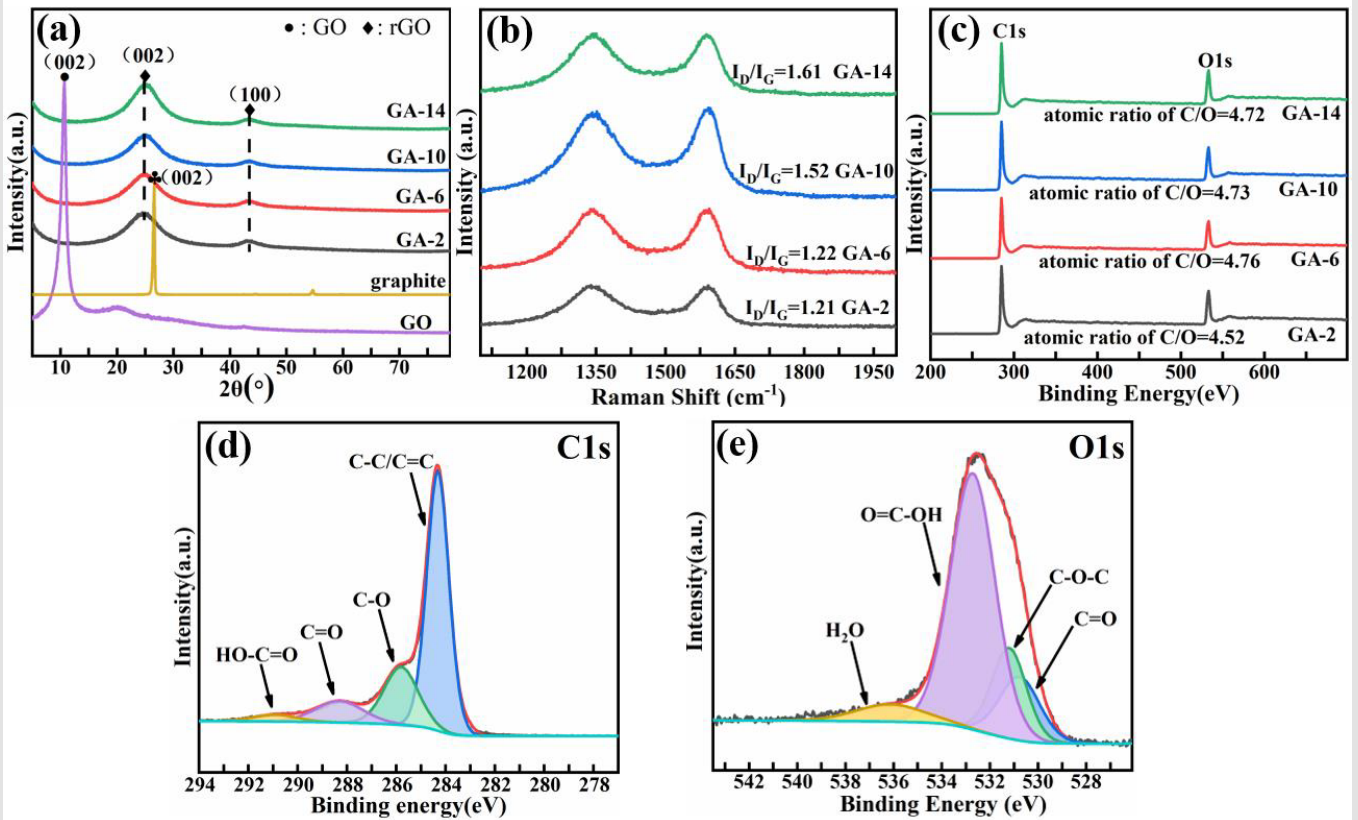

Figure 2: (a) XRD patterns; (b)Raman spectra; (c) XPS spectra of GA-X. High-resolution XPS spectra of (d) C 1s, and (e) O 1s in GA-6.

To further investigate the electrochemical properties of GA-X in LIBs, the corresponding samples are tested by the standard half-cell configuration. Shown in Figure 3(a) are the cyclic voltammogram curves (CVs) of the GA-6 electrodes with the first three cycles being measured at $0.2 \mathrm{mV} \mathrm{s}^{-1}$. In the first cycle, a sharp reduction peak at $0.25 \mathrm{~V}$ appears, accounting for the irreversible decomposition of electrolyte and the subsequent formation of a solid electrolyte interface (SEI) film on the surface of carbonaceous structure [3032]. In the subsequent cycles, CV curves are closely overlapped, which proves that the GA-6 anode has stable cyclic performance during the insertion and de-insertion process [33].

The charge-discharge curves of GA- 6 composite materials in the $1^{\text {st }}, 2^{\text {st }}, 3^{\text {st }}$ cycles are displayed in Figure 3(b). The GA- 6 exhibits an initial charge and discharge capacity of 1744.4/976.8 $\mathrm{mAh} \mathrm{g}^{-1}$ and the Coulombic efficiency is $56.0 \%$ for LIBs, which far outweighs that of others $1380.4 / 762.0$ (55.2\%), $1509.9 / 809.8$ (53.6\%), and 1411.8/828.1 (58.6\%) $\mathrm{mAh} \mathrm{g}^{-1}$. The massive irreversible capacity loss for LIBs is caused by the SEI layer decomposition, irreversible reaction between $\mathrm{Li}^{+}$and surface functional groups and irreversible desorption of ultra-fine pores [31]. Shown in Figure 3(c) are the testing results about the rate performance of GA-X anode at diversified current densities from $50 \mathrm{~mA} \mathrm{~g}^{-1}$ to $2 \mathrm{~A} \mathrm{~g}^{-1}$. In addition, the corresponding specific capacities of GA-6 are 671.2, 453.1, 292.9, 238.4, 201.2, 168.9 and $113.8 \mathrm{mAh} \mathrm{g}^{-1}$, respectively, which can be observed in Figure 3(d). However, another three samples only exhibit specific capacities of 583.2, 384.3, 241.5, 188.4, 159.5, 133.8, and $89.7 \mathrm{mAh} \mathrm{g}^{-1}$ for GA-2; 572.5, 387.6, 240.3, 194.1, 170.9, 143.8 and $91.3 \mathrm{mAh} \mathrm{g}^{-1}$ for $\mathrm{GA}^{-10}$; 562.3, 366.8, 224.9, 187.8, 160.7, 133.8 and $83.8 \mathrm{mAh} \mathrm{g}^{-1}$ for $\mathrm{GA}^{-14}$, respectively. It should be noted that when returning to the current condition of $100 \mathrm{~mA} \mathrm{~g}^{-1}$, there is 
still a reversible capacity of $428.4 \mathrm{mAh} \mathrm{g}^{-1}$ in GA-6, higher than 355.8 (GA-2), $380.2\left(\mathrm{GA}^{-10}\right)$ and $353.3\left(\mathrm{GA}^{-14}\right) \mathrm{mAh} \mathrm{g}^{-1}$. The dramatically enhanced $\mathrm{Li}^{+}$storage capacity of GA-6 is mainly because the threedimensionally porous structure is capable of providing more $\mathrm{Li}^{+}$ion transport channels and electrical conductivity [34-36].

Shown in Figure 3(e) is the information on the cycling stabilities of the four samples, and the corresponding test was performed at $100 \mathrm{~mA} \mathrm{~g}^{-1}$. As the hydrothermal reaction time increases, GA- 6 (about $664.8 \mathrm{mAh} \mathrm{g}^{-1}$ ) exhibits a significantly enhanced cycling performance than GA-2 (around $499.9 \mathrm{mAh} \mathrm{g}^{-1}$ ) after 100 cycles. This may be ascribed to the reason that the hydrothermal time is too short, which leads to insufficient reduction and too many oxygen-containing functional groups. When the hydrothermal time continues to increase, the capacity decays again (around 560.7 and $577.6 \mathrm{mAh} \mathrm{g}^{-1}$ ) due to the excessive aging of graphene oxide. The results obviously indicates that the hydrothermal synthesis time exerts great impact on the electrochemical performance of the anode. Additionally, as shown in Figure 3(e), all GA anodes show nearly $100 \%$ coulombic efficiency, which further confirms that the cycling stability of the obtained GA materials is very high for LIBs (Figure 4).
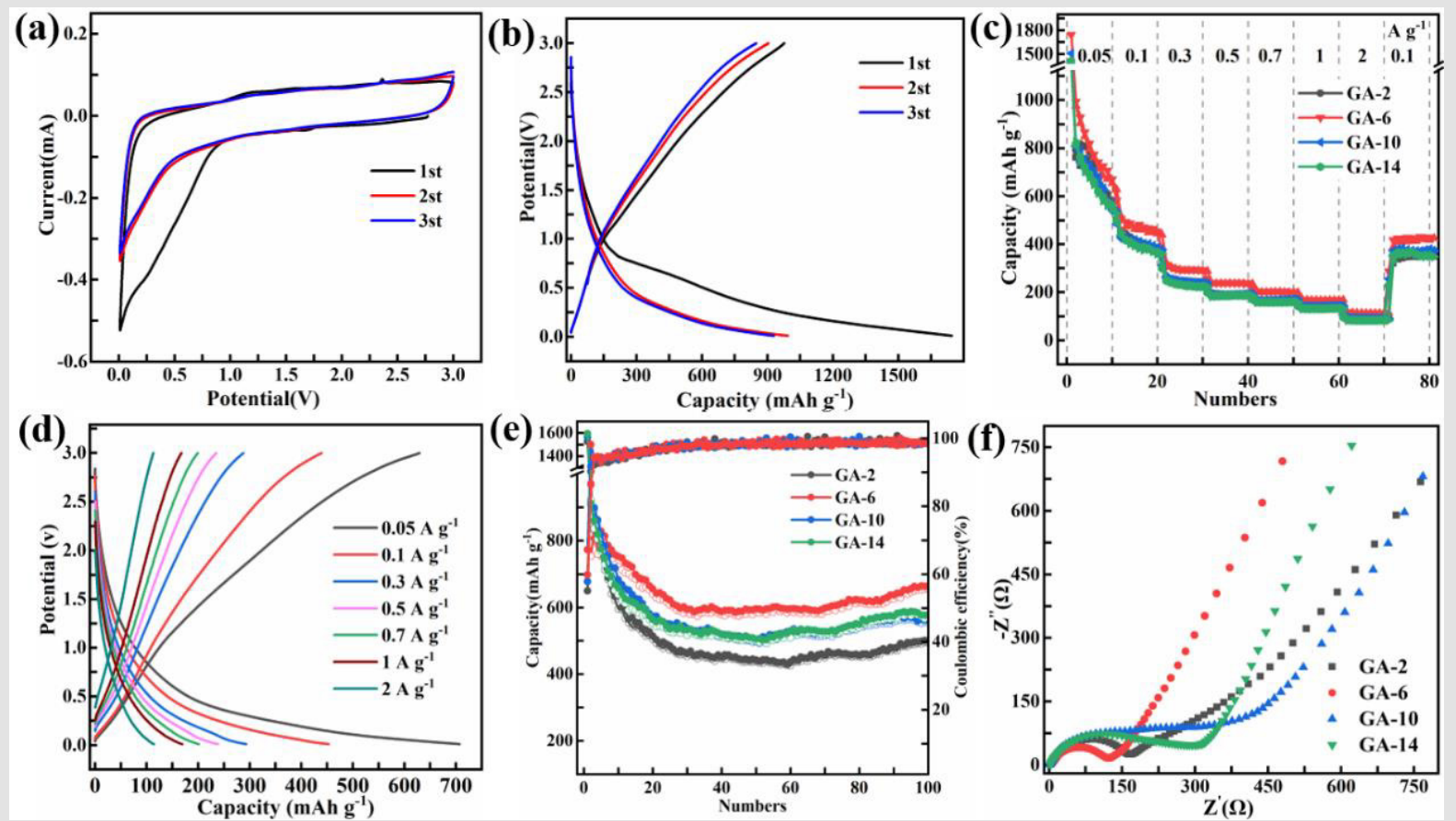

Figure 3: (a) CV curves of GA-6 at a scan rate of $0.2 \mathrm{mV} \mathrm{s}^{-1}$. (b) Charging/discharging of GA-6 at current density of $50 \mathrm{~mA} \mathrm{~g}^{-1}$. (c) Rate performances of GA-X electrodes. (d) The rate capabilities of GA-6 electrodes cycled at different current densities. (e) The cycling performance of GA-X electrodes in 100 cycles at $100 \mathrm{~mA} \mathrm{~g}^{-1}$. (f) Nyquist plots of the GA-X.

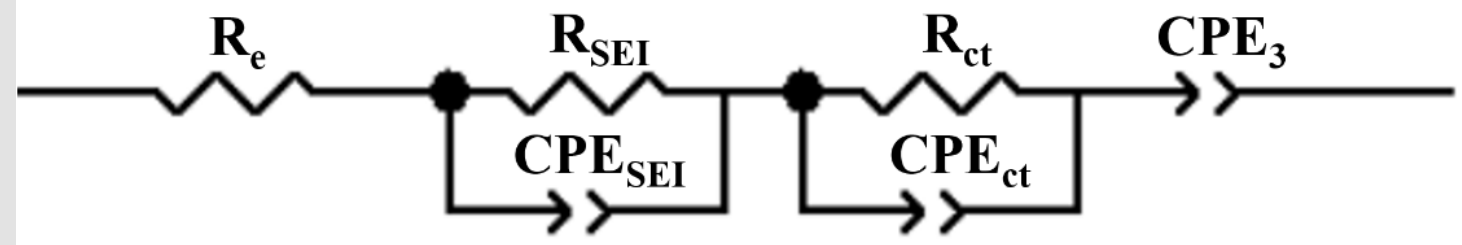

Figure 4: Nyquist plots of the electrodes.

The electrochemical impedance spectra (EIS) measurements are shown in Figure 3(f), which can further explain and verify the superior electrochemical performance. Nyquist plots of the composite illustrates an arc at the frequent regions corresponding to the interfacial charge-transfer resistance [37,38], and an approximate straight sloping line at the less frequent regions corresponding to Warburg impedance [39]. By contrast, GA-6 has a smaller semicircle and a higher slope, which is attributed to the porous structure preventing the self-aggregation of the GO sheets and providing more $\mathrm{Li}^{+}$ion diffusion channels. In addition, the GA-X 
samples have the same equivalent circuit fitting values (Table 2) and the result affirms that the GA- 6 composite possesses a smaller charge transfer impedance $\mathrm{R}_{\mathrm{SEI}}(100.3 \Omega)$ and Rct $(20.6 \Omega)$ than the GA-2 (150.8 $\Omega$ and $38 \Omega$ ), GA ${ }^{-10}(225.1 \Omega$ and $2.4 \Omega)$ and GA $^{-14}(135.7$ $\Omega$ and $176.8 \Omega$ ), which demonstrated that porous structure could decrease charge-transfer resistance and improve the electrical conductivity. In addition, to better quantitatively analyze the testing data, an ideal equivalent circuit is utilized for fitting the impedance spectra, as is displayed in Figure 4. Re denotes the resistance caused by the electrolyte, $\mathrm{R}_{\mathrm{SEI}}$ represents the resistance of the electrode surface film, Rct is the charge-transfer resistance and $\mathrm{CPE}_{\mathrm{SEI}}$ and CPEct are constant phase elements assigned as the surface film and double layer capacitance, respectively, and $\mathrm{CPE}_{3}$ is the constant phase element $[40,41]$.

Table 2: The detailed values of the equivalent circuit components used for fitting the experimental curve.

\begin{tabular}{|c|c|c|c|}
\hline Sample & $\mathbf{R e}(\boldsymbol{\Omega})$ & $\mathbf{R}_{\text {SEI }}(\boldsymbol{\Omega})$ & $\operatorname{Rct}(\boldsymbol{\Omega})$ \\
\hline GA-2 & 3.7 & 150.8 & 2.8 \\
\hline GA-6 & 1.9 & 100.3 & 20.6 \\
\hline GA-10 & 3 & 225.1 & 2.4 \\
\hline GA-14 & 2.7 & 135.7 & 176.8 \\
\hline
\end{tabular}

\section{Conclusions}

In summary, a simple yet effective hydrothermal approach was reported to synthesize self-assembled porous carbon framework (GA) with prestigious advantages of large surface area, specific porous structure, superior electronic conductivity, excellent mechanical characteristic and ultrafast electron transport kinetic. Benefiting from their surface defects and abundant porosity, the 3D porous graphene exhibits highly stable cycling performance (664.8 $\mathrm{mAh} \mathrm{g}^{-1}$ at $0.1 \mathrm{~A} \mathrm{~g}^{-1}$ after one hundred cycles). Moreover, it is convinced that by controlling the hydrothermal synthesis time, the surface defects of GA can be easily tailored. Correspondingly, GA-6 obtained by hydrothermal reaction for GA- 6 exhibits the highest electrochemical performance. The present work provides a simple strategy to the design of electrodes with high performance for LIBs.

\section{Acknowledgements}

This work was supported by the National Key Research Plan (2018YFB0604500), the National Natural Science Foundation of China (U1803114), the Key Scientific and Technological Project of Henan Province (202102210183), the China Postdoctoral Science Foundation (207500), Outstanding Foreign Scientists Studio of Coal Green Conversion of Henan Province (GZS2020012), National Natural Science Foundation of China (51974110) and the Program for Science \& Technology Innovation Talents in Universities of Henan Province (21HASTIT008).

\section{References}

1. Zhao H, Xing B, Zhang C, Huang G, Yu J, et al. (2020) MnOX-modified corrugated carton-derived hierarchical porous carbon with ultrafast kinetics behaviour for high-performance symmetric supercapacitors. Journal of Alloys and Compounds 848: 156423.

2. Zhao H, Xing B, Zhang C, Huang G, Liu Q et al. (2018) Efficient synthesis of nitrogen and oxygen co-doped hierarchical porous carbons derived from soybean meal for high-performance supercapacitors. Journal of Alloys and Compounds 766: 705-715

3. Zhong Y, Wu P, Ge S, Wu Y, Shi B, et al. (2020) An egg holders-inspired structure design for large-volume-change anodes with long cycle life. Journal of Alloys and Compounds 816: 152497

4. Wu Q, Jiang R, Liu H (2020) Carbon layer encapsulated $\mathrm{Fe}_{3} \mathrm{O}_{4} @$ Reduced graphene oxide lithium battery anodes with long cycle performance. Ceramics International 46(8): 12732-12739.

5. Fan X, Chen X, Dai L (2015) 3D graphene-based materials for energy storage. Current Opinion in Colloid \& Interface Science 20(5-6): 429438.

6. Blomgren GE (2016) The development and future of lithium-ion batteries. Journal of The Electrochemical Society 164(1): A5019.

7. Wu Y, Huang X, Huang L, Chen J (2021) Strategies for Rational Design of High-Power Lithium-ion Batteries. Energy \& Environmental Materials 4(1): $19-45$

8. Zhang L, Shi G (2011) Preparation of highly conductive graphene hydrogels for fabricating supercapacitors with high-rate capability. The Journal of Physical Chemistry C 115(34): 17206-17212.

9. Chen X, Han J, Lv X, Lv W, Pan Z, et al. (2019) Dense yet highly ion permeable graphene electrodes obtained by capillary-drying of a holey graphene oxide assembly. Journal of Materials Chemistry A 7(20): 12691-12697.

10. Chen K, Song S, Liu F, Xue D (2015) Structural design of graphene for use in electrochemical energy storage devices. Chemical Society Reviews 44(17): 6230-6257.

11. Shao JJ, Lv W, Yang QH (2014) Graphene: Self-Assembly of Graphene Oxide at Interfaces (Adv. Mater. 32/2014). Advanced Materials 26(32): 5732-5732.

12. Yi G, Li P, Xing B, Tian Q Zhang X, et al. (2021) Nitrogen-rich graphene aerogel with interconnected thousand-layer pancake structure as anode for high performance of lithium-ion batteries. Journal of Solid-State Chemistry 294: 121859.

13. Zhang J, Li C, Peng Z, Liu Y, Zhang J, et al. (2017) 3D free-standing nitrogen-doped reduced graphene oxide aerogel as anode material for sodium ion batteries with enhanced sodium storage. Scientific reports 7(1): 1-7.

14. Albarqouni YM, Lee SP, Ali GA, Ethiraj AS, Algarni H, et al. (2021) Facile synthesis of reduced graphene oxide aerogel in soft drink as supercapacitor electrode. Journal of Nanostructure in Chemistry 1-11.

15. Deng, Y, Luo C, Zhang J, Qiu D, Cao T, et al. (2019) Fast three-dimensional assembly of MoS 2 inspired by the gelation of graphene oxide. Science China Materials 62(5): 745-750.

16. Fan H, Yi G, Tian Q Zhang X, Xing B, et al. (2020) Hydrothermal-template synthesis and electrochemical properties of Co304/nitrogen-doped hemisphere-porous graphene composites with 3D heterogeneous structure. RSC Advances 10(60): 36794-36805.

17. Tao Y, Kong D, Zhang C, Lv W, Wang M, et al. (2014) Monolithic carbons with spheroidal and hierarchical pores produced by the linkage of functionalized graphene sheets. Carbon 69: 169-177. 
18. Wang J, Shi Z, Fan J, Ge Y, Yin J, et al. (2012) Self-assembly of graphene into three-dimensional structures promoted by natural phenolic acids. Journal of Materials Chemistry 22(42): 22459-22466.

19. Liu LL, Zhang HJ, Li S, Yang C, Yang PX (2015) On the Chemical Reduced Large Specific Surface Area Graphene Oxide and its Electrochemical Performances. In Applied Mechanics and Materials 723: 615-618.

20. Islam MM, Subramaniyam CM, Akhter T, Faisal SN, Minett AI, et al. (2017) Three-dimensional cellular architecture of sulfur doped graphene: selfstanding electrode for flexible supercapacitors, lithium ion and sodium ion batteries. Journal of Materials Chemistry A 5(11): 5290-5302.

21. Jiang Z, Zhang C, Qu X, Xing B, Huang G, et al. (2021) Humic acid resinbased amorphous porous carbon as high rate and cycle performance anode for sodium-ion batteries. Electrochimica Acta 372: 137850.

22. Xiao L, Wu D, Han S, Huang Y, Li S, et al. (2013) Self-assembled Fe203/ graphene aerogel with high lithium storage performance. ACS applied materials \& interfaces 5(9): 3764-3769.

23. Ferrari AC, Meyer JC, Scardaci V, Casiraghi C, Lazzeri M, et al. (2006) Raman spectrum of graphene and graphene layers. Physical review letters 97(18): 187401

24. Li R, Li J, Qi K, Ge X, Zhang Q, et al. (2018) One-step synthesis of 3D sulfur/nitrogen dual-doped graphene supported nano silicon as anode for Li-ion batteries. Applied Surface Science 433: 367-373.

25. Tang F, Jiang T, Tan Y, Xu X, Zhou Y (2021) Preparation and electrochemical performance of silicon@ graphene aerogel composites for lithium-ion batteries. Journal of Alloys and Compounds 854: 157135.

26. Byon HR, Gallant BM, Lee SW, Shao-Horn Y (2013) Role of oxygen functional groups in carbon nanotube/graphene freestanding electrodes for high performance lithium batteries. Advanced functional materials 23(8): 1037-1045.

27. Hu C, Zhai X, Liu L, Zhao Y, Jiang L, et al. (2013) Spontaneous reduction and assembly of graphene oxide into three-dimensional graphene network on arbitrary conductive substrates. Scientific reports 3(1): $1-10$.

28. Ye M, Dong Z, Hu C, Cheng H, Shao H, et al. (2014) Uniquely Arranged Graphene-on-Graphene Structure as a Binder-Free Anode for HighPerformance Lithium-Ion Batteries. Small 10(24): 5035-5041.

29. Sahoo M, Sreena KP, Vinayan BP, Ramaprabhu S (2015) Green synthesis of boron doped graphene and its application as high-performance anode material in Li ion battery. Materials Research Bulletin 61: 383-390.

\section{ISSN: 2574-1241}

DOI: 10.26717/BJSTR.2021.38.006205

Guiyun Yi, Yuanfeng Wu. Biomed J Sci \& Tech Res

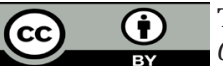

This work is licensed under Creative Commons Attribution 4.0 License

Submission Link: https://biomedres.us/submit-manuscript.php
30. Kang W, Lin B, Jiang Z, Liu Z, Feng L, et al. (2019) $\mathrm{Bi}_{2} \mathrm{O}_{2} \mathrm{CO}_{3}$ microspheres anchored on reduced graphene oxide nanosheets as electrode material for lithium-ion batteries and supercapacitors. Materials Letter 240: 299302 .

31. An SJ, Li J, Daniel C, Mohanty D, Nagpure S, et al. (2016) The state of understanding of the lithium-ion-battery graphite solid electrolyte interphase (SEI) and its relationship to formation cycling. Carbon 105: 52-76.

32. Han J, Kong D, Lv W, Tang DM, Han D, Zhang C, et al. (2018) Caging tin oxide in three-dimensional graphene networks for superior volumetric lithium storage. Nature communications 9(1): 1-9.

33. Zhang E, Jia X, Wang B, Wang J, Yu X, et al. (2020) Carbon dots@rGO paper as freestanding and flexible potassium-ion batteries anode. Advanced Science 7(15): 2000470.

34. Wang B, Ruan T, Chen Y, Jin F, Peng L, Zhou Y, et al. (2020) Graphenebased composites for electrochemical energy storage. Energy storage materials 24: 22-51.

35. Yu M, Zhang J, Li S, Meng Y, Liu J (2016) Three-dimensional nitrogen doped holey reduced graphene oxide framework as metal-free counter electrodes for high performance dye-sensitized solar cells. Journal of Power Sources 308: 44-51.

36. Wei W, Yang S, Zhou H, Lieberwirth I, Feng X, et al. (2013) 3D graphene foams cross-linked with pre-encapsulated Fe304 nanospheres for enhanced lithium storage. Advanced materials 25(21): 2909-2914.

37. Xing B, Zeng H, Huang G, Zhang C, Yuan R, et al. (2019) Porous graphene prepared from anthracite as high-performance anode materials for lithium-ion battery applications. Journal of Alloys and Compounds 779: 202-211.

38. Pei M, Wu Y, Qi Z, Mei D (2020) Synthesis and electrochemical performance of $\mathrm{NiO} / \mathrm{Fe} 3 \mathrm{O}_{4} / \mathrm{rGO}$ as anode material for lithium-ion battery. Ionics 26(8): 3831-3840.

39. Zeng H, Xing B, Zhang C, Chen L, Zhao H, et al. (2020) In situ synthesis of $\mathrm{MnO}_{2}$ /porous graphitic carbon composites as high-capacity anode materials for lithium-ion batteries. Energy \& Fuels 34(2): 2480-2491.

40. Lv Q, Song R, Wang B, Zhu H, Xu J, et al. (2020) Three-dimensional nitrogen-doped graphene aerogel toward dendrite-free lithium-metal anode. Ionics 26(1): 13-22

41. Tan C, Cao J, Khattak AM, Cai F, Jiang B, et al. (2014) High-performance tin oxide-nitrogen doped graphene aerogel hybrids as anode materials for lithium-ion batteries. Journal of Power Sources 270: 28-33.

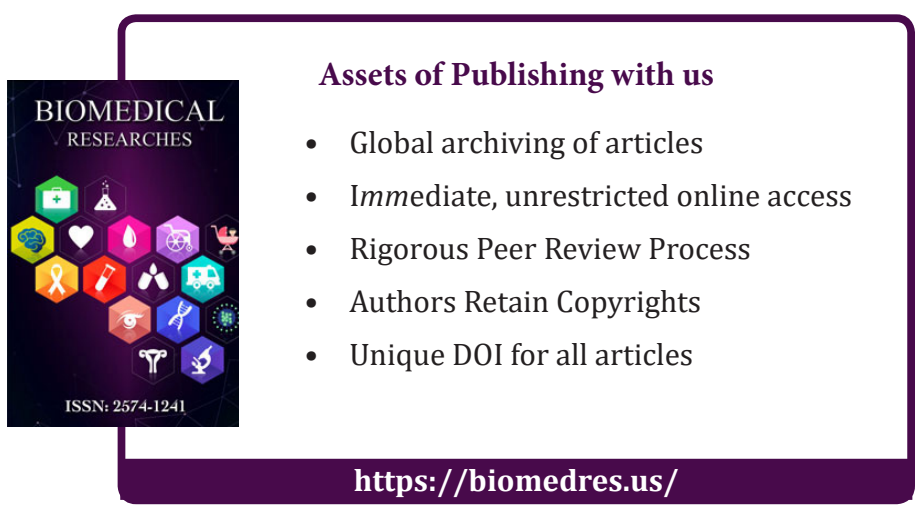

Copyright@ Guiyun Yi, Yuanfeng Wu | Biomed J Sci \& Tech Res | BJSTR. MS.ID.006205. 